\title{
Working with Amateur Astronomers
}

\section{Stephen J. Edberg}

International Halley Watch, Jet Propulsion Laboratory, California Institute of Technology, 4800 Oak Grove Drive, Pasadena, CA 911909-8099, U.S.A.

\section{Introduction}

Amateur astronomers have a vast store of talent and expertise in making astronomical observations. When carefully channelled, their enthusiasm coupled with these characteristics can lead to the acquisition of large volumes of high-quality astronomical data. The Amateur Observation Network of the International Halley Watch (IHW) was organized to encourage comet observations by amateurs, standardize techniques whenever possible, and then collect and archive these observations for use by the astronomical community in the near and distant future as part of the whole IHW archive. The lessons learned from this experience will be useful to organizers who plan observation campaigns involving amateur astronomers in the future.

\section{Background}

The IHW was first conceived by Louis Friedman of the Jet Propulsion Laboratory (JPL). From its conception, the IHW has included the observations of amateur astronomers in its campaign plans (Brandt et al., 1980).

In its final form, the IHW consisted of eight professional disciplines defined primarily by their use of astronomical technologies:

1. Large-Scale Phenomena (tail behaviour and morphology)

2. Near-Nucleus Studies (coma morphology)

3. Spectroscopy and Spectrophotometry

4. Astrometry

5. Photometry and Polarimetry

6. Meteor Studies

7. Radio Studies

8. Infrared Photometry and Spectroscopy

Amateur astronomers have the potential to make contributions to the first six disciplines, though a handful of the more naive wanted to try "ham" radio bouncing off Halley's Comet and one wondered about doing infrared observations. Ultimately, data for use in these six areas were received, ranging from large numbers of pho- 
tographs and visual magnitude estimates from many observers to a single set of photoelectric observations from one observer.

Organizing amateur observation was more easily accomplished on the basis of technique, rather than technology. Six areas of effort were identified:

1. Visual Observations, including magnitude estimates and drawings

2. Photography, from short to long focal lengths

3. Astrometry

4. Spectrophotography

5. Photoelectric Photometry

6. Meteor Observations, including hourly counts, photography and spectrophotography

To standardize amateurs' efforts in these areas the International Halley Watch Amateur Observers' Manual For Scientific Comet Studies (Edberg, 1983) was prepared and published. Besides being distributed through the U.S. Superintendent of Documents, two private publishers reprinted it and it was translated or adapted to Chinese, Finnish, French, German, Hungarian, Italian, Japanese, Polish and Spanish by interested parties outside the United States.

For each of the areas of amateur study a chapter was devoted to detailing the correct observing procedure to use, based on a literature search and consultation with experienced observers. Alternate methods were sometimes presented. Required calibration techniques were included. Sometimes data analysis methods were also presented so observers could analyze their own work.

Besides an IHW registration form, standard data report forms requesting many specifics were included in the manual. These were set up to be understood without reference to the manual, if necessary. Space was allocated for presentation of the data, method used for obtaining them, and standardization or calibration techniques used. In areas such as photographic processing, a standard practice simply could not be imposed so the report form asked for details of the observer's personal procedures.

To better keep registered observers as well as the whole community of amateur astronomers informed, the IHW Amateur Observer's Bulletin was published and distributed free, courtesy of the Planetary Society. Circular letters were also used occasionally to inform registered observers of important events, updates in report forms, or in data reporting procedures.

The IHW ultimately coordinated observing campaigns on Comet $\mathrm{P} /$ Crommelin $(1984 \mathrm{IV}=1983 \mathrm{n})$, Comet P/Giacobini-Zinner $(1985 \mathrm{XIII}=1984 \mathrm{e})$, and Comet P/Halley (1982i, no Roman numeral designation as yet). Crommelin served as a trial-run target, to test the IHW archiving procedures (see Sekanina and Aronsson, 1985). Comet Giacobini-Zinner (G-Z) was added to the IHW program when the International Sun-Earth Explorer No.3 (ISSE-3) spacecraft was diverted to G-Z and renamed the International Cometary Explorer (ICE). 


\section{Observations on the Observers}

Over 1400 amateur astronomers registered with the Amateur Observation Network. In addition, well over 200 contributors sent in observations by no registration form (the majority of these were meteor observers). There were a total of about 1000 data contributors, ranging from beginner to very experienced.

In terms of observing technique it was, in a few cases, more difficult to get experienced observers to observe IHW requirements than novices. Many experienced amateur observers have difficulty in maintaining an unbiased, scientific attitude about their results or their methods of obtaining them. In the area of magnitude estimation there were difficulties in getting observers to agree on what standard comparison stars to use and that poor conditions of atmospheric transparency could affect their results. In a few cases, non-IHW reporting methods made their data difficult to enter in the IHW computer archive.

The amateur community, as a group, does not have a good understanding of the scientific method and the need for calibration. This was reflected, especially among photographers, by their failure to supply requested calibration photographs of selected astronomical targets. Perhaps too much was expected of contributors.

In order to include more than a tiny fraction of the submitted observations, the author has felt it necessary to relax many of the data reporting and calibration requirements and to prepare all the reasonable data for archiving. A strong warning will go with the archive, though, that data users must be selective about what they use and how they use it.

\section{Observations on the Observations}

The following table summarizes the data from amateur astronomers on the IHW's three campaign comets. The text following the table includes discussions of astrometry, photoelectric photometry, and meteor observations which are not listed in the table.

IHW Data Archives

\begin{tabular}{lllll}
\hline Comet & $\begin{array}{l}\text { Number of Observations } \\
\text { Visual Observations }\end{array}$ & \multicolumn{2}{c}{ Photographs } & Spectra \\
& Mag. Ests. & Drawings & \\
P/Crommelin & 197 & 0 & 0 & 0 \\
P/Giacobini-Zinner & $1000^{*}$ & $50^{*}$ & $50^{*}$ & 0 \\
P/Halley & $10000^{*}$ & $600^{*}$ & $2000^{*}$ & 52 \\
& $*$ estimated number & & & \\
\hline
\end{tabular}

The IHW data archives on G-Z and Halley will include a small number of copies on magnetic tape, a widely distributed collection of printed volumes, and a version on compact optical disk. The Crommelin archive was produced on magnetic tape and printed versions (Sekanina and Aronsson, 1985). 
Visual Observations - Magnitude Estimates. The large number of Halley observations allow a number of interesting studies to be made. Edberg and Morris (1986) concluded that scatter in Halley's visual light-curve is most strongly influenced by observer experience and that experienced observers tend to see a larger coma and make correspondingly brighter magnitude estimates. Coma morphology also affects estimates but the instrument used has much less effect on estimates than these other factors. Inexperienced observers show a marked tendency towards estimate precisions of only $1 / 2$ magnitude, whereas experienced observers are often precise to $1 / 10$ magnitude. Much more investigation will be possible when the complete computer archive is available for analysis.

Visual Observations - Drawings. The drawings varied in quality from simple sketches of a comet's position in a star field to careful renderings of fine detail in the coma. Selected drawings may be included in the printed archive for comparison with professional images there. All suitable drawings will be catalogued in the archives. Little detailed study has been done as yet on the drawings.

Photography. Photographs, especially in the case of Halley, were made with focal lengths ranging from a few centimeters to over 3.5 meters. Both black \& white and color emulsions were used. All photographs showing useful detail will be listed in the archives and amateur photographs filling gaps in professional coverage will be archived with the professional disciplines.

Astrometry. At the initiation of the IHW a small number of amateur astronomers were already making astrometric observations of comets and had established their reputations for high-quality measurements well enough to work directly with the professional discipline specialists. Newcomers to the field submitted their work to the Amateur Observation Network, which in turn checked those results with the professional discipline specialists. Regrettably and not surprisingly the data from novices were not usable. The residuals of their positions were returned to these observers along with a note of encouragement that their work would improve with practice.

Spectrophotography. Four observers submitted 52 Halley spectra. These low-dispersion spectra, obtained with a variety of instruments, will be listed in the archive.

Photoelectric Photometry. The very few of these observations made by amateur astronomers will be forwarded to the professional Photometry and Polarimetry discipline network for their ultimate disposition.

Meteor Studies. Hundreds or even thousands of hours of meteor counts have been made by over 300 observers. Often these were made in only one or two hour stretches, while a longer interval is more desirable. Two meteor photographs have been received. All these data have been forwarded to the professional Meteor Studies discipline specialist team for analysis and disposition to the archive. 


\section{Conclusion}

Amateur astronomers can make significant contributions to observational campaigns using several methods of study. The organizers of such campaigns must understand the motivations and limitations of their amateur contributors and design the program accordingly. Early in the planning process, leading amateur observers in the campaign topic should be consulted on how best to organize the amateur community and on the methods of observation used by amateurs. Those contacts should be maintained throughout the campaign. Detailed instructions should be issued to the observers, but the organizer must be prepared for the unexpected.

\section{Acknowledgements}

Hundreds of amateur astronomers have contributed to make the IHW Amateur Observation Network a success. Mikael Aronsson, Carlo de Antonio, Mary Firth, and Charles Morris have all assisted in the data preparation or analysis. The research described in this paper was carried out by the Jet Propulsion Laboratory, California Institute of Technology, under contract with the National Aeronautics and Space Administration.

\section{References}

Brandt, J.C., Friedman, L.D., Newburn, R.L., and Yeomans, D.K., eds., 1980, The International Halley Watch - Report of the Science Working Group, Pasadena, NASA-JPL Publication TM $82181(400-88)$

Edberg, S.J., 1983, International Halley Watch Amateur Observers' Manual For Scientific Comet Studies, Pasadena, NASA-JPL Publication 83-16

Edberg, S.J. and Morris, C.S., 1986, "Observational Factors Affecting Studies of Comet P/Halley's Visual Light Curve" in Proc. ESLAB Symposium on the Exploration of Halley's Comet, Noordwijk, The Netherlands, ESA-ESTEC Publication SP-250, p. 609

Sekanina, Z and Aronsson, M., 1985, Archive Observations of Periodic Comet Crommelin, Pasadena, NASA-JPL Publication 86-2 